\title{
3D Simulation of navigation problem of people with cerebral visual impairment
}

\author{
Yahya Q. I. Al-Fadhili ${ }^{1}$, Paul W.H. Chung ${ }^{2}$, Baihua Li $^{3}$, Richard Bowman ${ }^{4}$ \\ ${ }^{1}$ Computer Science Dept., College of education, Mosul University, Iraq. \\ ${ }^{2,3}$ School of Science, Computer department, Loughborough University, UK. \\ ${ }^{4}$ Great Ormond Street Hospital, London, UK. \\ 1 y.q.i.al-fadhili@lboro.ac.uk \\ 2 P.W.H.Chung@lboro.ac.uk, 3 B.Li@lboro.ac.uk \\ 4 richard.bowman@gosh.nhs.uk
}

\begin{abstract}
Cerebral Visual Impairment (CVI) is a medical area that concerns the study of the effect of brain damages on the visual field (VF). People with CVI have difficulties in their mobility and they have behaviours that others find hard to understand due to their visual impairment. A branch of Artificial Intelligence (AI) is the simulation of behaviour by building computational models that help to explain how people solve problems or why they behave in a certain way. This paper describes a novel computational system that simulates the navigation problem that is faced by people with CVI. This will help relatives, friends, and ophthalmologists of CVI patients understand more about their difficulties in navigating their everyday environment.

The navigation simulation system is implemented using the Unity3D game engine. Virtual scenes of different living environment are also created using the Unity modelling software. The vision of the avatar in the virtual environment is implemented using a camera provided by the 3D game engine. Filters that mimic visual defects are created automatically and placed in front of the visual field of the avatar. The filters are based on the visual field charts of individual patients. Algorithms for navigation based on the limited vision have also been developed to demonstrate navigation problems because of the visual defects. The results showed different actions for the navigation behaviours according to the patients' vision, and the navigations differ from patient to another according to their different defects.
\end{abstract}

Keywords: vision impairment simulation, Cerebral Visual Impairment, imitation, AI based modeling and navigation.

\section{Introduction}

Cerebral Visual Impairment (CVI) is medically defined as a neurological disorder caused by damage to the occipital lobes and/or to the visual pathways and it is associated with disturbed visual sense because of the brain deterioration rather than eye damage [1, 2, and 3]. 
Visual perception is a function of the eyes and brain together, thus, it's the way that human knows and understands the world around them through what they see using both eyes and brain [4]. It is the process or ability by which sensory information from our sense eyes is transformed to produce the recognition of shape, size, and brightness of objects and the distance of how close or how far away an object is [5].

CVI affects a person's ability to see and recognize obstacles in their surrounding area, thus affect their ability to move around in their everyday environment. Therefore they often appear to be clumsy but it is due to their inability to see. There is the need to demonstrate the difficulties that CVI patients encountered so that their relatives and friends have a better understanding of the problems that they face in their everyday living. If the CVI patient is a child then the better understanding will also help teachers in schools to accommodate for their needs due to their vision deficiency.

This paper describes the design and implementation of a novel 3D virtual reality system that simulates the navigation problems faced by people with CVI. In this system an avatar will act as an individual moving around in a 3D environment and will avoid or bump into obstacles mimicking the behavior of the particular individual. The system is able to simulate the visual impairment of individual patients based on their visual field vision chart results. This serves to highlight the specific problems that individuals faced and how the environment may be adapted to suit their needs.

\section{Related work}

Several studies have provided evidence that CVI is possibly a contributor of cognitive and intellectual dysfunction. Therefore, CVI is considered as a disease that requires educational and training intervention. In 2008, Dutton [6] developed a set of strategies to help parents and teachers to support children with CVI in different daily situations.

Instead of coping strategies, there are other projects that develop devices that would help the blind or partially blind. For example, the Google Glass developed through the Open Glass Project [7] can be considered as a hands-free smartphone. It responds to voice commands by taking a picture in front then processes the image, searches for a specified object of interest and indicates whether it exists within view. This makes it easier for CVI patients to identify and locate objects in their living environment [8].

Another device called Badge3D is a tag detection system. Barcodes (tags) are attached to selected objects in the living environment with the tags clearly visible for the recognition system to work. The system was designed specifically for indoor use to help visually impaired patients navigate their living environment independently. Like Google Glass, the system is voice activated [9].

Another project, called Hazard Perception Test (HPT), focused on detecting what CVI patients can see with their visual defects. Films of driving scenarios are used to measure the rate of responses of new drivers for detecting hazards using a computerbased test. The test focused on superior (upper) and inferior (lower) visual field deterioration. The study concluded that hazard detection is more affected by superior than inferior defects [10]. 
Research has also attempted to simulate and measure the reading performance of glaucoma patients. Patients tend to use their central visual field to gaze at the line and the word being read. An eye tracker was adjusted or distorted to match the visual field of the reader, as the centre of the reader's eyes is not the centre of the visual field due to their visual impairment. Eye movements were video recorded. The gazing position was shown by a red point over each word being read. The blur of the peripheral areas in the scene indicates the defects corresponding to visual field areas [11].

\section{$3 \quad$ Aim and overall architecture}

The aim of this project is to develop algorithms that simulate and illustrate what the CVI patients can see and how they would navigate with their vision impairment using a 3D modelling environment. Figure 1 shows the main steps of how the system is accomplished and the input and output for each sub-process.

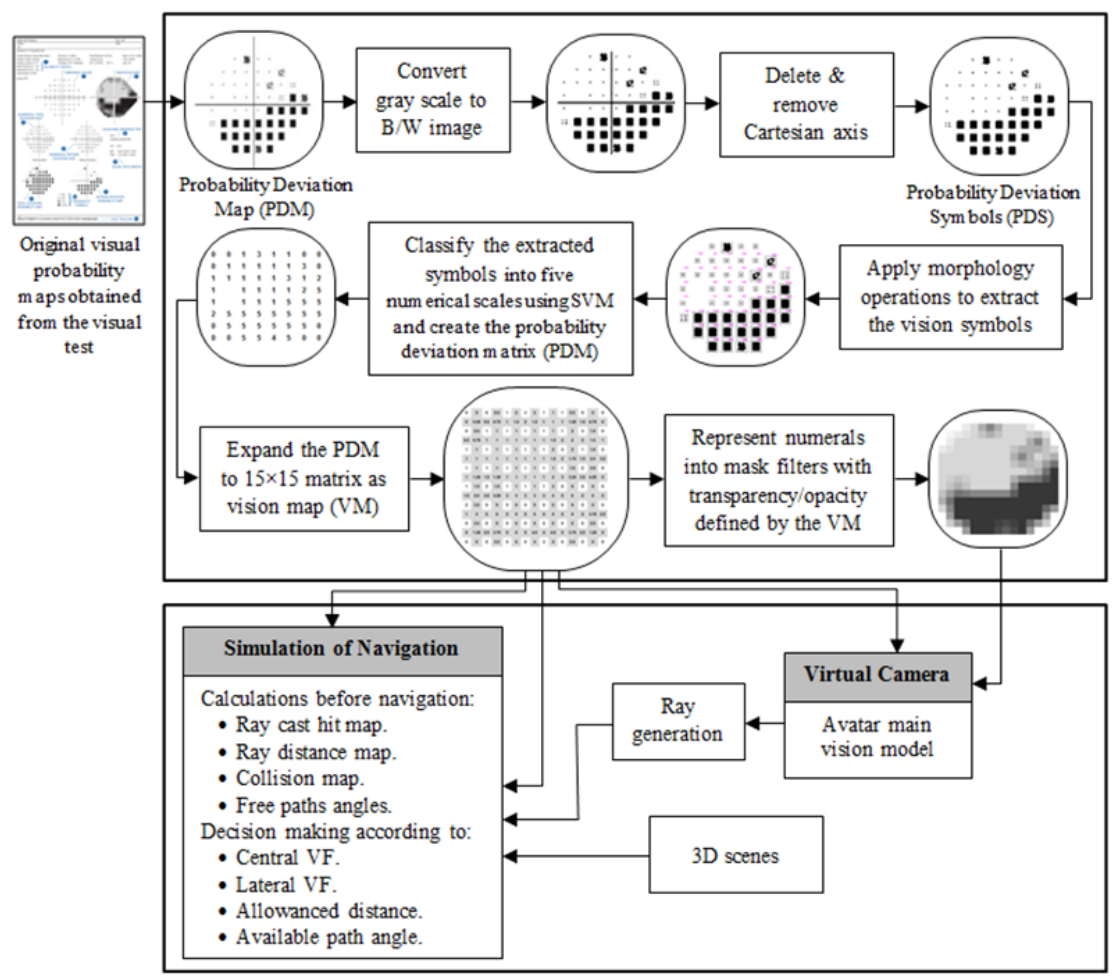


Fig. 1. System architecture - Cerebral Visual Impairment simulation of navigation behaviour

\section{Methodology}

\subsection{Image pre-processing}

Visual field is tested using special device called perimetry. It produces a chart with many numeral, deviation and symbolical patterns that describe a person's vision. The last symbolic pattern in the chart which is the deviation probability map (DPM) represents the final calculations of the testing areas in a symbolic form. It consists of five scaled symbols and each represents the degree of the deficiency in specific visual spot.

Digitizing the image symbols of vision deficiency needs to be done first. This can be achieved by detecting the symbol region from the VF chart, and converting them into black \& white symbol block image (B/W). The existed Cartesian coordinates were located and deleted using the property of the largest connected component/biggest object; see Fig. 2(a). A sequence of mathematical morphology operations were used to specify shapes in this input image. The values represented by a detected vision symbol block were derived from the number of pixels, the centroid of the vision symbol and symbol area boundaries, see Fig. 2(b).

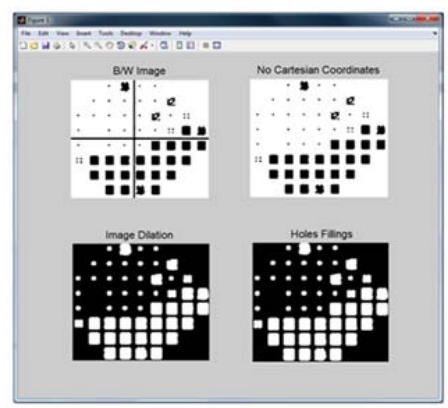

( a )

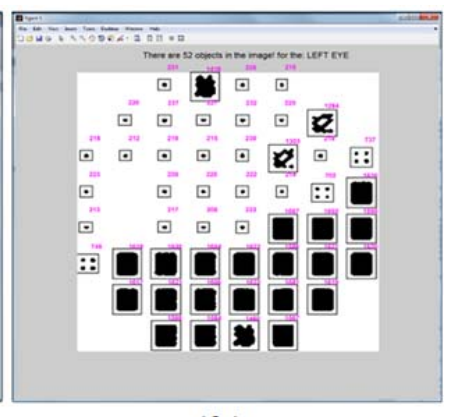

(b )

Fig. 2. (a) Image processing for features extraction and (b) object identification

The extracted symbols were saved as image patches individually. They were classified into five classes representing visibility levels using a supervised machine learning classifier support vector machine (SVM) [12, 13]. Training samples of visibility symbols were saved in five separated folders (representing the five classes) for learning and matching process as feature space. Then the extracted input symbols are classified and mapped to these output features. The result of classification is an integer number [1 - 5] refer to the degree of visual deterioration. These data are saved into a 2-D array of size $8 \times 8$. As shown in Fig. 3(b), the spots are digitized presenting significant visual differences via levels of transparency. The $8 \times 8$ array was expanded to $15 \times 15$; this is achieved by inserting other elements between two successive elements to smooth the transitions. Thus, the six gray scales $[0,1, \ldots, 5]$ are extended to 21 trans- 
parency levels $[0,0.25,0.5,0.75,1,1.25, \ldots, 4.25,4.5,4.75,5]$ as shown in fig. 3(c). The final numeral array of the clear and defect values represent the Vision Map which is considered as the main map that the proposed computational model depends on.

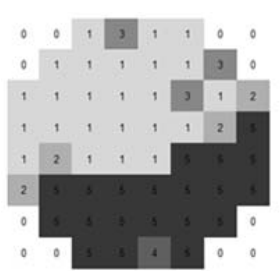

( a)

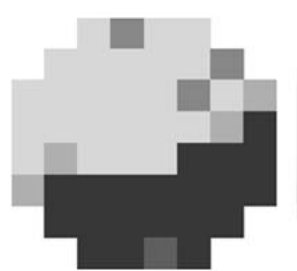

( b )

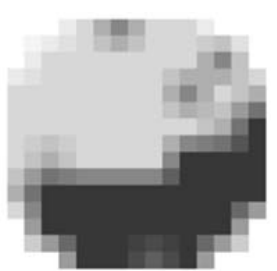

(c)

Fig. 3. Vision map: (a) classified six transparency levels in $8 \times 8$ vision map, (b) the discrete transition between transparency levels and (c) the expanded smoothed $15 \times 15$ vision map

Each visual level in the vision map was converted into a gray image with specified degree of transparency as a mask filter using Photoshop CS6. This image is in three channels with equal colour values; a fourth (alpha) channel was added for transparency using texture's advanced properties in Unity3D.

\subsection{The projection of the vision map}

As the brain damages affect patients' eyes, parts of the vision area are confused and the clarity/blurring of the vision area is determined by the deteriorating degree, and thus they determine the grade of transparency or blur that used. Unity3D is a 3D game engine which is suitable for projecting the CVI patients' vision into 3D. An avatar can be used to represent the character of a patient in First Person Shooter (FPS) mode. The attached camera to the top of the avatar can be used to simulate the patient's eyes. Therefore it was used to project the mask filters onto it to make the game view window in Unity reflect the VF of the patient to clarify what this patient is really see.

A $15 \times 15$ array of plane objects was designed and centralized in front of the main camera to cover the whole vision view. Each one has a numeric value taken from the vision map. A specific transparent texture was attached to every plane programmatically and automatically. The filters mask moves within the avatar camera and project the blurred spots in the person's eyes during its navigation to emulate defect vision. Unity has basic functions and features that provide 3D physics to the avatar and other assets in the scene. Such physics include colliders and ray-casting. The project considered the vision rays as beams that start from the eye and reach any point in the 3D space. Therefore, vision rays were generated using the ray-casting physics feature to simulate vision beams. A second array $(15 \times 15)$ of planes also was designed and placed in front of the mask filter. These planes were gathered into one object to represents the rays object. The lengths of each ray depend on the numerical value taken from the vision map and produce the Ray Map. The bigger values (means high percentage of opacity) are represented by short beam in length. While small values (means high percentage of transparency) are represented by long beam. The avatar 
moves ahead and turns away depending on the movement and turning of the attached rays object according to a main algorithm script. Figure 4 shows the normal vision rays instantiation and representation, while Fig. 5 clarifies the deteriorated vision.

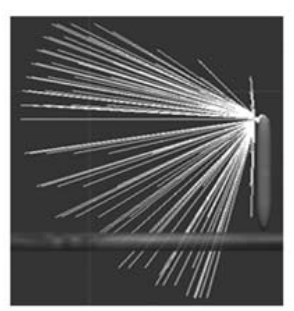

( a )

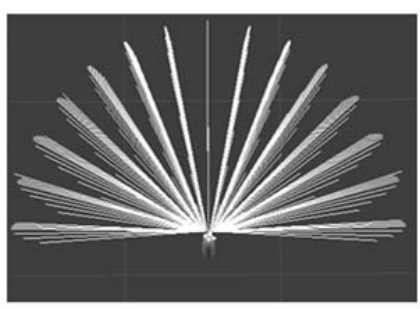

(b)

Fig. 4. Vision rays of normal vision, (a) side view and (b) upper view
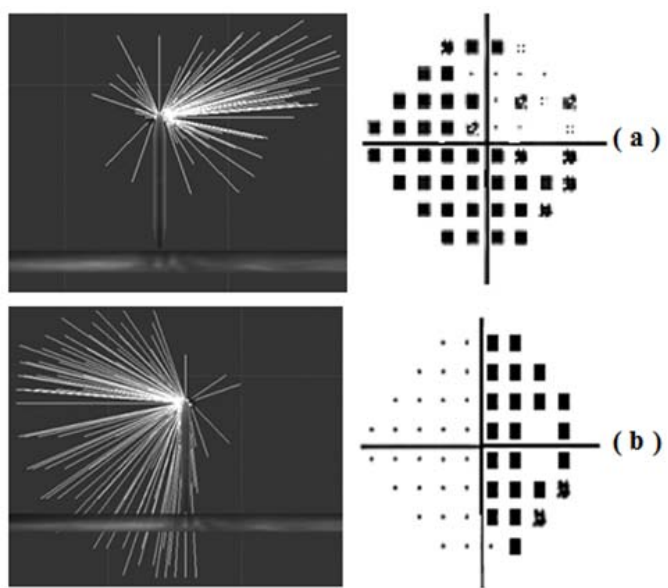

Fig. 5. Vision rays for deteriorated visibility (back views), (a) left superior and most of the interior VF defects and (b) right side VF defect

By using this, it facilitates the control of the avatar navigation according to whether the rays hit (colliding) or not. The length of each ray is calculated as follows:

$$
\begin{gathered}
\text { Ratio }=\text { View }_{\text {depth }} / 5 \\
\text { Ray }_{\text {length }}=\text { View }_{\text {depth }}-\text { Defect }_{\text {level }} * \text { Ratio }
\end{gathered}
$$

Where ratio is the percentage of the view depth to the largest scale in the pattern, view $_{\text {depth }}$ is the depth of the person vision, and defect $t_{\text {value }}$ is the scale value extracted and calculated from the pattern.

According to the VF measurements for central, nasal and lateral fields [14], each ray was instantiated according to the attached C\# script to each plane and rotated about the $\mathrm{x}$ and $\mathrm{y}$ axes according to the $\mathrm{i}^{\text {th }}$ and $\mathrm{j}^{\text {th }}$ of their positions in the vision map. The rotation was calculated as follows: 


$$
\begin{gathered}
y_{\text {rotation }}=(j \times 12.857)-90 \\
x_{\text {rotation }}=(i \times 4.286)-30 \quad \text { for superior (upper) field } \\
x_{\text {rotation }}=(i \times 10.714)-75 \quad \text { for inferior (lower) field }
\end{gathered}
$$

where $y_{\text {rotation: }}$ the rotation about $\mathrm{y}$-axis.

$x_{\text {rotation: }}$ the rotation about $\mathrm{x}$-axis.

\subsection{Simulation of navigation}

The ray map shows the areas and the surrounding objects that can be seen or not. The hit-rays mean objects are detected; however, the non-hit rays mean objects are not detected. This principle facilitates the navigation through a flat platform like rooms, going upstairs or downstairs, and recognizing moving objects depending on the vision rays only.

According to the vision map array and the generated ray map array, a new $(15 \times 15)$ array that holds the generated ray physics were created representing the Raycast Map array. During the navigation and at each updated frame, the rays will hit or collide with the objects in the environment producing Ray Distance Map array. All maps are to be dependent on for further calculation during the navigation process. The fifteen columns of rays were rotated about y-axis from $+90^{\circ}$ to $-90^{\circ}$ according to their position producing fifteen rotating angles. These fifteen angles will be considered as pathways angles. If the avatar turns away from any visible obstacle, therefor a decision will be made based on the most suitable of the fifteen angles and the clearest half of vision.

As vision, in fact, relies on the central vision field in the first place then relies on the lateral vision field, the navigation algorithm depends on this fact. Therefore, it starts checking the central field for the obstacles in the front paths. The lateral vision can be relied on selecting the proper angle for turning away and to avoid the objects on sides like walls.

The ratio representing the percentage of the view depth to the largest scale in the pattern is calculated. Then the two halves of the vision map values are counted to indicate and select which half of the vision is clearest and to be used for turning decision.

The navigation behaviour goes through three stages:

- Stationary situation: the real person (or avatar) starts navigation from a stopping situation. Even when it is moving, there is a tiny period of time when it transfers its position from step to step. This time is considered as stopping or pausing time. The stationary stage gives an enough time to the navigator to reason the surroundings.

- Reasoning situation: where the person recognizes the surroundings then necessary measurements is calculated to make a decision and take appropriate actions.

- Action situation: it's when the person (or avatar) decides what action to take. These actions can be step forward, stop and wait, or step backward. 
These reasoning processes include the procedures of creating and calculating different maps. Therefore the avatar at each updated frame calculates and checks all maps as reasoning before taking the normal action which is to step forward.

\section{$5 \quad$ Implementation and results}

A projection code was attached to the main camera in Unity to open and load the file of numeral values of VF and distribute them to corresponding planes. Then, a desired transparency texture is selected accordingly to project each visual area into specific position on the camera.

Results were taken from a set of VF patterns so that each pattern represents a visual deterioration. Figure 6 shows the original patterns of the PDM symbols taken from three VF chart, and the projected filters for the defect symbols onto a 3D world scene. The above first projection explores a person with clear and normal vision. While the middle projection displays the VF for a totally inferior with almost superior visual deteriorations. But the last projection displays the VF for right-side eyes deterioration.
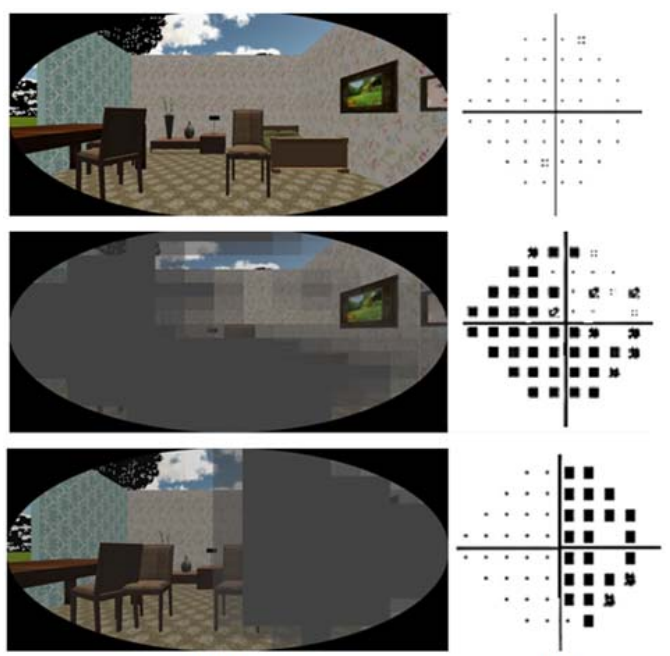

( a )

(b)

Fig. 6. (a) Simulation of visual impairment-projected VF patterns in a 3D scene and (b) Original VF patterns

Three VF charts were projected in the scene and the navigation algorithm on them was executed. For a normal vision person, navigation simulation showed the expected behaviour as a normal person would act. Figure 7 shows the expected behaviour of normal VF person facing an obstacle. The performed actions of the avatar were matched what a real person would act. The images in this figure show a top view of the avatar navigation with its vision rays casting. 


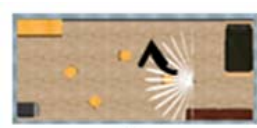

(1)

Chair is detected Right turn expected

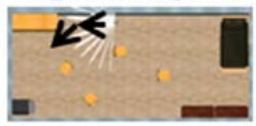

(4)

Wardrobe is detected Left turn expected

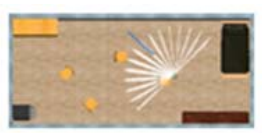

(2)

Right turn performed

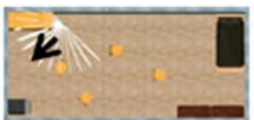

(5)

Left turn performed

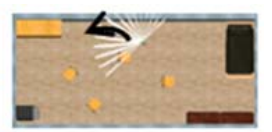

(3)

Wall is detected Left turn expected

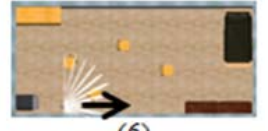

(6)

Central VF is clear

Go forward expected

Fig. 7. The expected and the obtained behaviour for a person having normal vision

Refer to Fig. 5(b), another scenario for a right-side eye defect VF pattern was implemented, see Fig. 8. The camera view window which represents what the avatar sees is attached with the navigation view window representing the top view of the scene looking at the avatar to show what this child exactly sees. This will justify the reason of his behaviour and actions during his navigation. In Fig. 8 (4), when a wall is detected, the avatar would not turn to the left (its best vision half) because a left side walk indicator is set. So, the avatar would turn to the right even the right side is totally unseen. This simulates the visually impaired person when he/she expects a path to the unseen side and turn away from it while his best visible side is facing obstacles.

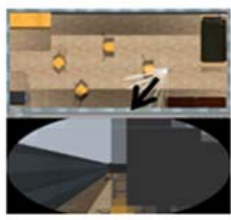

(1)

Chair is detected Left turn expected

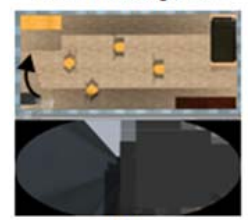

(5)

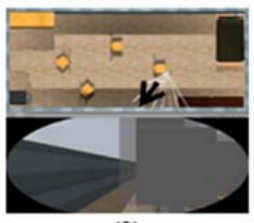

(2)

Left turn performed

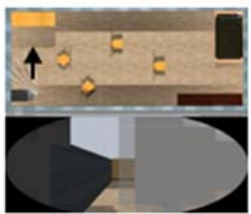

(6)

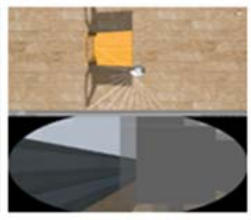

(3)

Chair is no more seen Slight bump expected

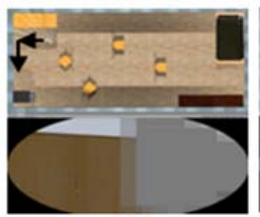

(7)

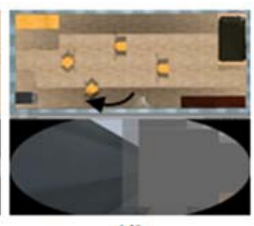

(4)

Wall is detected Right turn expected

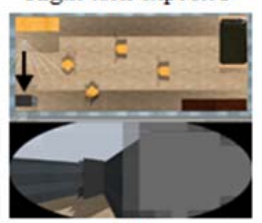

(8)

Right turn expected

Right turn performed

Wardrobe is detected

Left turn performed Left turn expected

Fig. 8. The expected and obtained behaviour for a person having defects in the right side of the eye

In Fig. 9, the simulation was executed on a situation of a child has the defects in the most of his VF, see figure 5(a). Only few spots in his right superior VF are clear. This person and as expected, he will bump with many obstacles but the walls and high objects during his navigation. 


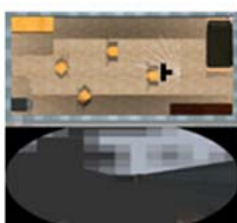

(1)

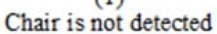

Bump expected

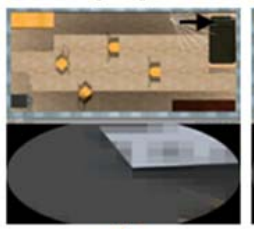

(5)

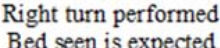

Bed seen is expected

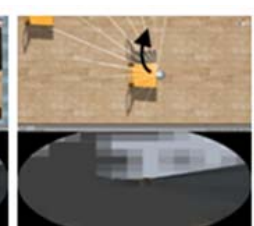

(2)

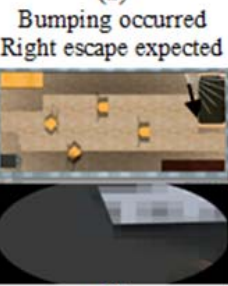

(6)

Bed is detected Right turn expected

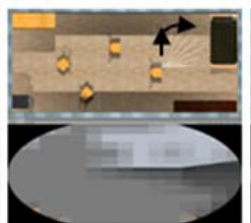

(3)
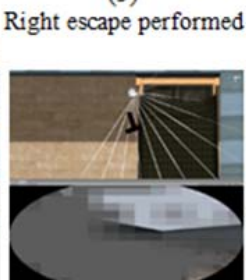

(7)

Bed is no more seen Bump expected

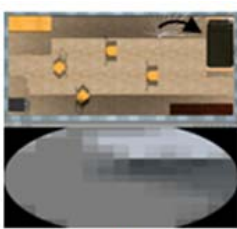

(4)

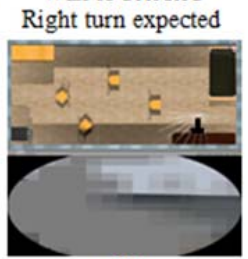

(8)

Drawers is not seen Bump expected

Fig. 9. The expected and obtained behaviour for a person having defects in the inferior and left superior

\section{Summary and Conclusions}

The probability symbols of vision in the VF chart can be extracted and projected into $3 \mathrm{D}$ world to simulate visual impairment behaviours. The visibility regions in the projected world can be presented via different levels of texture transparency representing the impairment areas of vision defects. According to the value of visibility, a visual ray can be initiated, drawn and rotated for visual areas. Thus, the whole created rays will represent a zone of vision beams and can be used to guide navigation behaviors.

The objects in the surroundings can be seen when these rays hit them, while the objects that are not touched by the rays are considered not visible to the avatar. The ray hit and collision properties can facilitate and clarify how the patient can avoid obstacles, and/or how he avoids bumping into them. The coding algorithms can be set according to the normal human navigate behaviour.

The result of applying the algorithms on different VF charts showed different decisions for the turning away process. These decisions were taken depending on the better part of vision, walkable route and the existence of the lateral obstacles.

The current work is to project the 3D scene and the visual impairment defects using the HTC VIVE headset so that people without visual field impairment can experience for themselves what it is like to move around with visual field defects. Experiments are also planned to assess the behavior exhibit by people using the HTC VIVE against the avatar simulation. 


\section{Acknowledgment}

This work was implemented during $\mathrm{Mr}$ Yahya Al-Fadhili was a $\mathrm{PhD}$ student at Loughborough University, UK. The authors thank the Iraqi (MOHESR) for the scholarship funding.

\section{References}

1. Bernas-Pierce J., Pollizzi J., Altmann C., Lee B., Hoyt C. (1998). Cortical visual impairment paediatric visual diagnosis fact sheet. SEE/HEAR. Blind Babies Foundation.

2. Huo R., Burden S.K., Hoyt C.S., Good W.V. (1999). Chronic cortical visual impairment in children: aetiology, prognosis, and associated neurological deficits. $\mathrm{Br} J$ Ophthalmol. 83(6): 670-675.

3. Dutton G.N., Saeed A., Fahad B., et al. (2004). The association of binocular lower vision field impairment, impaired simultaneous perception, disordered visually guided motion and inaccurate saccades in children with cerebral visual dysfunction- a retrospective observational study. Eye. 18: 27-34.

4. Swift S.H., Davidson R.C. and Weens L.J. (2008). Cortical impairment in children: presentation, intervention, and prognosis in educational settings. TEACHING Exceptional Children Plus. 4(5), Article 4.

5. Isak L. Nilsson, William V. Lindberg (2008). Visual Perception: New Research. Nova Science Pub Incorporated.

6. Dutton G.N. (2008). Strategies for dealing with visual problems due to cerebral visual impairment. Scottish Sensory Centre.

7. Duffy M. (2013, May 1). Google Glass Applications for Blind and Visually Impaired Users. VisionAware Blog.

8. Owano N. (2013, August 14). OpenGlass apps show support for visually impaired. PHYSORG

9. Iannizzotto G., et al. (2005). Badge3d for Visually Impaired. IEEE Computer Society Conference on Computer Vision and Pattern Recognition (CVPR'05).

10. Glen F.C., Smith N.D. and Crabb D.P. (2014). What types of visual field defects are hazardous for driving? ARVO Annual Meeting Abstracts. 2014.

11. Crabb D.P. (2014). Personal Communication.

12. Vapnik N. (1998). Statistical Learning Theory. New York, John Wiley \& Sons, 421-422.

13. Cortes C. and Vapnik N. (1995). Support-vector-networks. Machine Learning. 20(3):273.

14. Walker H.K., Spector R.H., Hall W.D., Hurst J.W., editors. (1990). Chapter 116. Visual Fields. In: Clinical Methods: The History, Physical, and Laboratory Examinations. 